\title{
DETERMINACIÓN DE UN ELEMENTO DE VOLUMEN REPRESENTATIVO DE PROBETAS DE TABLERO TENSADO
}

\section{DETERMINATION OF A REPRESENTATIVE VOLUME ELEMENT OF STRESS-LAMINATED DECK SPECIMENS}

\author{
Daniela Sepúlveda ${ }^{1}$, William Gacitúa ${ }^{2}$, Cecilia Bustos ${ }^{2 \star}$, Peter Dechent ${ }^{3}$, Alain Cloutier ${ }^{4}$
}

\section{RESUMEN}

El objetivo de este estudio fue definir un elemento de volumen representativo para caracterizar las propiedades mecánicas de probetas de tableros tensados de Pinus radiata D. Don y de Eucalyptus nitens. La determinación del elemento de volumen representativo permitió identificar la sección más pequeña de las probetas que presenta las particularidades de este material compuesto y por consiguiente, representa a la probeta en su conjunto. Para esto, las propiedades nanomecánicas de las paredes celulares y lamela media fueron determinadas por nanoindentaciones. Este estudio se desarrolló en dos etapas. En la primera etapa, el anillo de crecimiento representativo de las piezas de madera bajo compresión perpendicular a la fibra, fue determinado. En la segunda etapa, el tablón representativo de la probeta de tablero tensado sometido a compresión a largo plazo, fue determinado. Los resultados de la primera etapa, mostraron que no existe una tendencia específica de las propiedades nanomecánicas a través de los anillos de crecimiento en la sección transversal de las piezas de Pinus radiata y Eucalyptus nitens bajo compresión perpendicular a la fibra. En la segunda etapa, se estableció que los esfuerzos de compresión a largo plazo en la probeta de tablero tensado principalmente afectaron las propiedades nanomecánicas de la estructura celular de los tablones exterior y central de la probeta. Lo anterior permitió establecer que el elemento de volumen representativo de una probeta de tablero tensado se localizó en la madera temprana del anillo de crecimiento más alejado a la médula en la sección transversal del tablón central.

Palabras claves: Propiedades mecánicas, compresión perpendicular, nanoindentaciones, Pinus radiata, Eucalyptus nitens.

\begin{abstract}
The aim of this study was to define a representative volume element to characterize the mechanical properties of stress-laminated deck specimens of radiata pine and Eucalyptus nitens. The determination of the representative volume element allowed to identify the smallest representative section of the stress-laminated deck. For this, the nanomechanical properties of the cell walls and middle lamellas were determined by nanoindentations. This study was conducted in two phases. In the first phase, the growth ring representative of the pieces of wood under compression perpendicular to the grain was determined. In the second phase, the board representative of the stress-laminated deck specimens subjected to long-term compression loads and variable environmental conditions was determined. The results obtained in the first phase of the study showed that there was no specific tendency of the nanomechanical properties through growth rings in the cross-section of Pinus radiata

\footnotetext{
${ }^{1}$ Estudiante de Magíster en Ciencia y Tecnología de la Madera. Universidad del Bío-Bío. Concepción. Chile. dasepul@alumnos.ubiobio.cl

Autor para correspondencia: ^cbustos@ubiobio.cl

Recibido: 16.12. 2011 Aceptado: 13.05. 2012
}

${ }^{2}$ Profesor Asociado. Departamento de Ingeniería en Maderas. Facultad de Ingeniería. Universidad del Bío-Bío. Concepción. Chile. wgacitua@ubiobio.cl

${ }^{3}$ Profesor Asociado. Universidad de Concepción. Concepción. Chile. pdechen@udec.cl.

${ }^{4}$ Profesor Titular. Universidad Laval. Quebec. Canadá. Alain.Cloutier@sbf.ulaval.ca.
\end{abstract}


and Eucalyptus nitens specimens subjected to compression loads perpendicular to the grain. In the second phase of the study, it was found that long-term compression loads in stress-laminated deck specimens mainly affect the mechanical properties of the cellular structure of exterior and central boards. Finally, the representative volume element in a stress-laminated deck specimen was found in the earlywood of the growth ring farthest to the pith in the cross section of the central board.

Keywords: Mechanical properties, compression perpendicular, nanoindentation, Pinus radiata, Eucalyptus nitens.

\section{INTRODUCCIÓN}

En Chile se han introducido variadas especies forestales, entre las cuales destacan los cultivos de Pinus radiata D. Don y Eucalyptus nitens. Por un lado, el pino radiata es la principal especie comercial de uso estructural en el país, con una superficie del 63\% del total de bosques plantados (INFOR 2010, Fritz 2004). No obstante, esta especie se considera poco durable, por lo que en la mayoría de sus usos requiere ser protegida con preservantes. Por otro lado, los bosques de Eucalyptus nitens alcanzan un $7 \%$ de la superficie total de bosques de plantación, siendo la tercera especie más plantada en Chile (INFOR 2010). Su aplicación se concentra principalmente en la industria de celulosa. Esta especie posee tasas de crecimiento que van de $30 \mathrm{a} 45 \mathrm{~m}^{3} /$ ha al año, haciéndola muy propensa al agrietamiento (INFOR 2004).

En coníferas y en latifoliadas se manifiestan diferencias en su estructura anatómica en las direcciones transversal y longitudinal, que son atribuidas a modificaciones en el cambium, factores genéticos y factores ambientales (Diaz-vaz 2003, Panshin y De Zeeuw 1980). Asimismo, debido a la heterogeneidad y anisotropía de la madera, las propiedades físicas y mecánicas cambian según la dirección en que éstas se midan. Estos aspectos originan que el comportamiento físico y mecánico de la madera sea variable. El comportamiento mecánico de este tipo de material puede ser estudiado con ayuda de un elemento de volumen representativo (RVE) a microescala, que sea capaz de representar cuantitativamente las características a macroescala. Los RVEs han sido utilizados en modelaciones mecánicas de las propiedades elásticas de materiales poliméricos y de la microestructura de la madera (Valavala et al. 2009, Astley et al. 1997). En ambos estudios, el RVE fue una buena aproximación de las propiedades medidas experimentalmente.

La nanoindentación es una herramienta que permite evaluar el comportamiento mecánico de las células de la madera a nivel de nanoescala. Al igual que en las propiedades mecánicas obtenidas a macroescala, la nanoindentación también muestra diferencias en estas propiedades entre las células de madera tardía y temprana, siendo las propiedades de estas últimas aproximadamente un 50\% menor que las células de madera tardía (Eder et al. 2009, Cramer et al. 2005, Wimmer et al. 1997). Por su parte, Wimmer et al. (1997), al estudiar la estructura celular de abeto rojo (Picea rubens), lograron establecer que las capas de la pared celular poseen distintas propiedades mecánicas debido a los cambios en la composición química de éstas.

La estructura celular de la madera, sus constituyentes poliméricos y la disposición estructural a diferentes niveles de microestructura dan a la madera un comportamiento complejo en el uso de ésta en obras civiles, más aún si ésta se expone a la acción de cargas externas con variaciones de temperatura y contenido de humedad. En particular, cargas mayores de compresión perpendicular originan una densificación y rigidización de las fibras debido a la deformación y el aplastamiento de la pared celular que se producen en la dirección de la solicitación (Navi y Heger 2005). Los tableros tensados de madera utilizados en puentes son un ejemplo de aplicación estructural de este tipo de comportamiento, dado que las piezas de madera son tensadas en dirección transversal por medio de barras de acero (Figura 1). Existen investigaciones en esta área informadas por Figueroa (2011), 
Ugalde (2004) y Muñoz (2002) que estudiaron el comportamiento reológico de probetas de tableros tensados de madera. El principal interés en este tipo de estructuras es la pérdida de capacidad de carga en el tiempo producida por variaciones en el tensado de las barras, que pueden ser controladas a través de retensados al tablero (Ritter 1990).

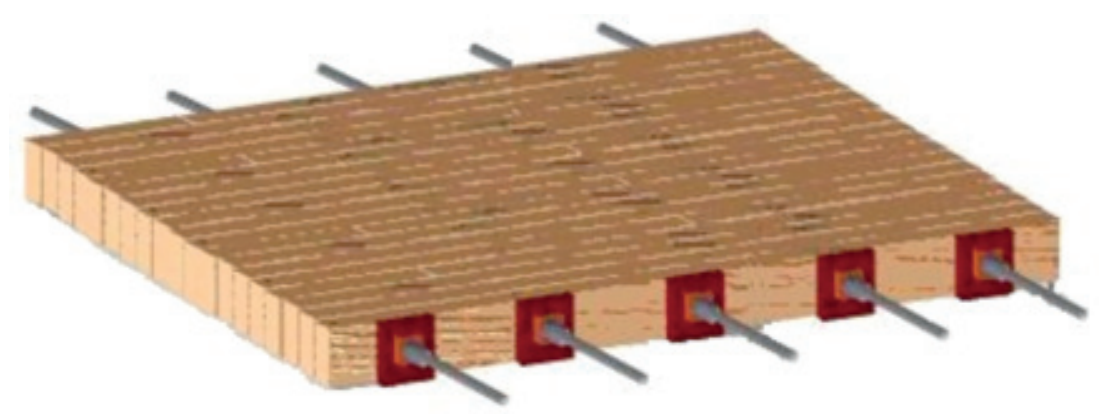

Figura 1. Esquema de un tablero tensado de madera sólida (Muñoz 2002).

La mayoría de estos trabajos están basados en el análisis del comportamiento estructural a macroescala. Sin embargo, no existe información sobre el comportamiento estructural de la madera bajo cargas y condiciones ambientales variables a nivel ultraestructural que permita relacionarla con el comportamiento a macroescala. De este modo, el objetivo general de este estudio fue definir el elemento de volumen representativo de probetas de tablero tensado a través del análisis de las propiedades nanomecánicas, como módulo de elasticidad, dureza, factor de creep y factor de relajación, de la estructura celular de Pinus radiata y Eucalyptus nitens, usando nanoindentaciones.

\section{MATERIALES Y MÉTODOS}

\section{Materia Prima}

El estudio se llevó a cabo con madera de Pinus radiata D. Don clasificada estructuralmente como C24, según norma BS EN 338 (2003) y con madera de Eucalyptus nitens proveniente de la zona de Yungay, VIII Región. La madera de pino radiata presentó una densidad básica promedio de $0.493 \mathrm{~g} /$ $\mathrm{cm}^{3}$ y un contenido de humedad promedio de $11 \%$, mientras que la densidad promedio de la madera de Eucalyptus nitens fue de $0.484 \mathrm{~g} / \mathrm{cm}^{3}$ y su contenido de humedad promedio de $15 \%$.

El estudio se desarrolló en dos etapas, donde en la primera se definió el anillo de crecimiento representativo de piezas de Pinus radiata y Eucalyptus nitens sometidas a compresión perpendicular a la fibra. En la segunda, consistió en definir el tablón representativo de la probeta de tablero tensado de pino radiata que estuvo bajo compresión a largo plazo y a condiciones ambientales variables, aunque controladas.

\section{ETAPA 1}

\section{Obtención y preparación de las probetas sometidas a compresión}

De una pieza de madera de $4.1 \times 14.1 \times 20 \mathrm{~cm}^{3}$ se obtuvieron 3 probetas de $4.1 \times 5 \times 5 \mathrm{~cm}^{3}$ por cada especie. Dos de estas probetas fueron sometidas a compresión perpendicular a la fibra (plano LT) durante 8 horas en una máquina de ensayo universal Instron de capacidad 5 ton (Figura 2), bajo niveles de carga de 250 y $500 \mathrm{~kg}$, equivalentes a 1 y $2 \mathrm{MPa}$ de presión específica sobre la madera, respectivamente. Estos valores corresponden al 50\% y 100\% del nivel de tensado que se aplicó en la 
probeta de tablero de madera sólida utilizada en la etapa 2. La tercera probeta fue utilizada como control sin aplicarle carga de compresión.

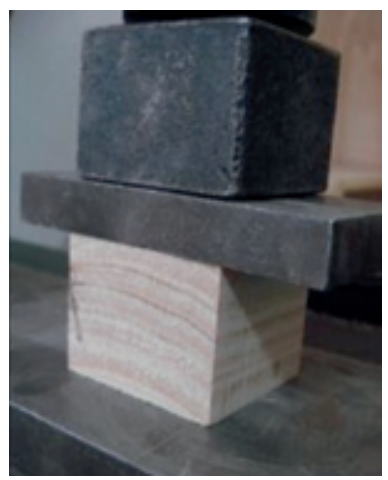

Figura 2. Probeta de pino radiata sometida a compresión perpendicular en máquina de ensayo universal.

\section{Preparación de las muestras para la determinación de las propiedades nanomecánicas}

De las muestras sometidas a compresión y de la muestra control se extrajeron cubos de $3 \mathrm{~mm}$ por lado, para cada anillo de crecimiento de la sección transversal, considerando madera temprana y tardía. Los cubos fueron cortados una vez que la carga de compresión fue retirada. Se procedió a identificar cada anillo de crecimiento y el tipo de madera (Figura 3), obteniendo un total de 10 muestras para las probetas de Pinus radiata y 5 muestras para las probetas de Eucalyptus nitens. Los cubos fueron obtenidos desde listones de $1 \times 1 \mathrm{~cm}^{2}$ luego, se llevaron a dimensiones finales de $3 \mathrm{~mm}$ por lado mediante cuchillos de acero de ultrafilo. Los cubos fueron llevados a condición anhidra y fueron impregnados con resina epoxi para darle soporte mecánico a la estructura celular. Con un micrótomo se dejó la superficie transversal libre de rugosidades, para ello se trabajó con un cuchillo de vidrio y luego con uno de diamante. Finalmente, las muestras fueron colocadas en una placa metálica para la medición de sus propiedades nanomecánicas.

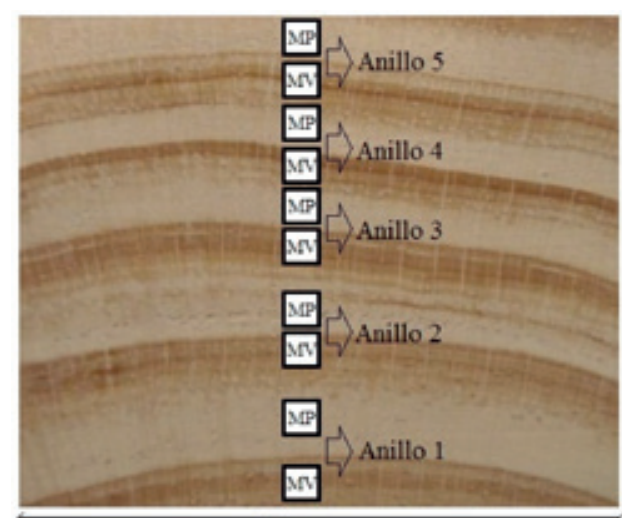

$5 \mathrm{~cm}$.

(a)

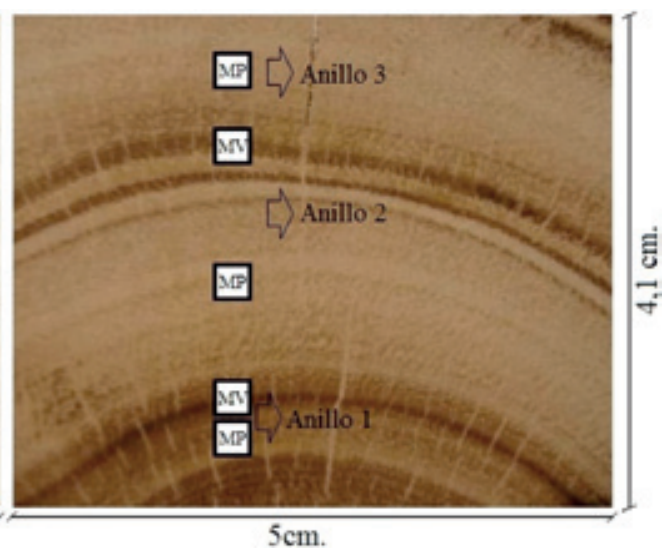

(b)

Figura 3. Anillos de crecimiento en sección transversal de probetas de (a) Pinus radiata y (b) Eucalyptus nitens, (MV: madera tardía, MP: madera temprana). 


\section{Propiedades nanomecánicas}

A través de nanoindentaciones se determinó el módulo de elasticidad $\left(E_{s}\right)$ y la dureza $(H)$ de la lamela media y de la capa S2 en la pared celular de ambas especies, utilizando un nanoindentador TriboIndenter TI-900 marca Hysitron, con una punta de diamante piramidal tipo Cube Corner con sus caras orientadas en $90^{\circ}$, y un ciclo de carga máxima de $100 \mu \mathrm{N}$ (Figura 4).

El valor de $E_{s}$ se determinó con la expresión (1) (Fischer-Cripps 2004):

$$
E_{s}=\left(1-v_{s}^{2}\right)\left(\frac{1}{E_{r}}-\frac{1-v_{i}^{2}}{E_{i}}\right)^{-1}
$$

$E_{i}$ es igual a $1140 \mathrm{GPa}$ con una razón de Poisson de 0.07 para la punta de diamante. $E_{r}$ fue obtenido directamente del ensayo. Se consideró una razón de Poisson igual a 0.3 para las muestras de pino radiata y Eucalyptus nitens (Salmén 2004).

La dureza se obtuvo del ensayo a través de la expresión (2):

$$
H=\frac{P_{\max }}{A_{h c}}
$$

donde $P_{\max }$ es la carga máxima de indentación y $A_{h c}$ es el área de contacto proyectada, que es una función de la profundidad de contacto.

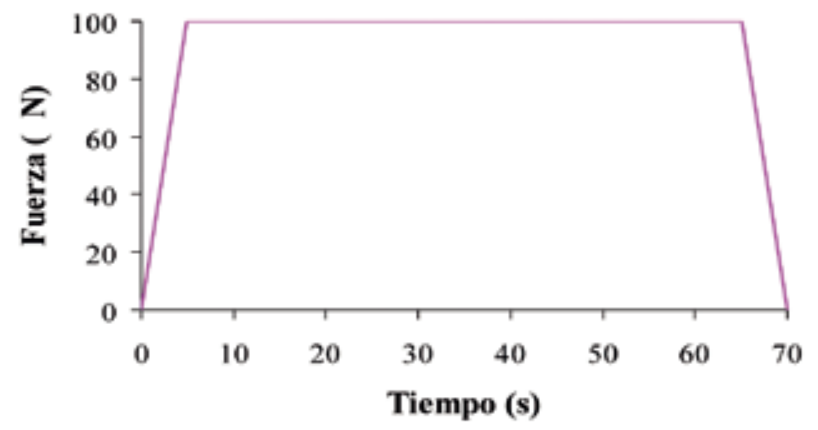

Figura 4. Ciclo de carga con fuerza constante utilizado en las nanoindentaciones para la determinación de $E_{s}$ y $\mathrm{H}$.

Las nanoindentaciones se realizaron en una superficie de $10 \times 10 \mu \mathrm{m}^{2}$ y de $20 \times 20 \mu \mathrm{m}^{2}$, en muestras de pino radiata y de Eucalyptus nitens y, respectivamente, realizando un mínimo de 5 nanoindentaciones en la pared celular y 3 en la lamela media.

\section{Análisis estadístico}

Un análisis de varianza (ANOVA) de tres factores se utilizó para estudiar el efecto del nivel de carga, anillo de crecimiento y tipo de madera (Tabla 1) en las propiedades de $E_{s}$ y $\mathrm{H}$ de la pared celular y lamela media en Pinus radiata y Eucalyptus nitens, con un nivel de significancia $\alpha=0.01$ dado que no hubo repeticiones. El procedimiento MIXED ajustado a modelos lineales mixtos del 
programa SAS fue utilizado con sentencias arbitrarias. Las comparaciones pareadas se realizaron con el test protected Fisher LSD.

Tabla 1. Diseño experimental general en el análisis del efecto de compresión perpendicular.

\begin{tabular}{|c|c|c|}
\hline Variables & Pinus radiata D. Don & Eucalyptus nitens \\
\hline Nivel de carga & 0 & 0 \\
(MPa) & 1 & 1 \\
& 2 & 2 \\
\hline \multirow{2}{*}{ No anillo de $_{\text {crecimiento }}$} & 1 & 1 \\
& 2 & 2 \\
\hline \multirow{2}{*}{ Tipo de madera } & 4 & 3 \\
& 5 & Temprana \\
& Temprana & Tardía \\
\hline
\end{tabular}

\section{ETAPA 2}

\section{Armado y acondicionamiento de la probeta de tablero tensado}

Se confeccionó una probeta de tablero tensado con madera de pino radiata clasificada estructuralmente C24, según norma BS EN 338 (2003). La probeta se comprimió en dirección normal a la fibra por medio de barras de acero con una carga máxima inicial de $2 \mathrm{MPa}$, utilizando un sistema de anclaje en los bordes laterales con placas de acero. La probeta se armó con 8 tablones dispuestos uno sobre otro por las caras de las piezas. Las dimensiones de cada tablón fueron de $4.1 \times 14.1 \times 20 \mathrm{~cm}^{3}$. Cada tablón fue identificado con las letras A, B, C y D, con el fin de establecer la posición relativa de éstos con respecto del borde de la probeta (Figura 5). El historial de carga fue determinado mediante el uso de strain gauges. El armado de la probeta se realizó al interior de una cámara de clima, de dimensiones $4.6 \times 4.8 \mathrm{~m}^{2}$, y se acondicionó durante 190 días, con variaciones de temperatura entre $20^{\circ} \mathrm{C}$ y $5^{\circ} \mathrm{C}$, y de humedad relativa (HR) entre $65 \%$ y $85 \%$ (Figura 6 ).

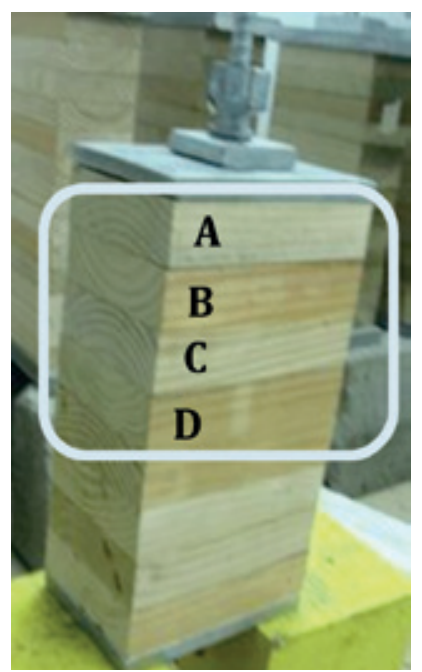

Figura 5. Probeta de tablero tensado de pino radiata mostrando la identificación y la posición de los tablones que la constituyen. 


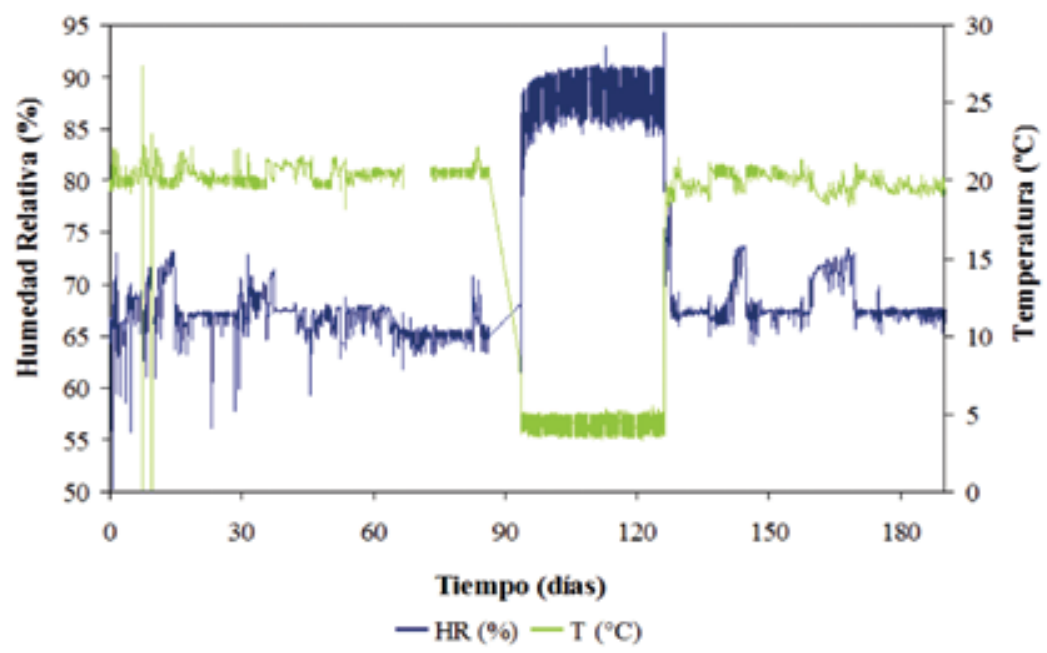

Figura 6. Historial de condiciones ambientales en cámara de clima durante 190 días.

Un tablón, identificado con la letra E, sin compresión y acondicionado a las mismas temperaturas y HR que la probeta de tablero tensado, sirvió para utilizarlo como muestra control.

\section{Preparación de las muestras para la determinación de las propiedades nanomecánicas}

Se analizaron cuatro de los ocho tablones que componen la probeta de tablero tensado, tablones A, B, C, D y el control (E) (Figura 5). Para ello, la probeta de pino radiata fue desarmada al interior de la cámara de clima y de cada tablón se obtuvo dos cubos de $3 \mathrm{~mm}$ de lado para los ensayos de nanoindentación, una de ellas con células de madera temprana y la otra con células de madera tardía, que en conjunto corresponden al anillo de crecimiento más alejado de la médula en su sección transversal. La preparación de estos cubos se realizó utilizando el mismo procedimiento de la etapa 1.

\section{Propiedades nanomecánicas}

Se determinaron las propiedades a nanoescala del módulo de elasticidad $\left(E_{s}\right)$, dureza $(\mathrm{H})$, factor de creep y factor de relajación en la pared celular y en la lamela media. $E_{s}$ y $\mathrm{H}$ se determinaron según lo señalado en la etapa 1 . El factor de creep $C$ se determinó por el ciclo de fuerza constante indicado en la etapa 1 (Figura 4) y de acuerdo a la ecuación (3).

$$
C=\frac{h_{2} \square h_{1}}{h_{1}} \times 100
$$

donde $h_{1}$ y $h_{2}$ son la profundidad de la indentación para cuando se alcanzó el nivel de fuerza constante y para cuando se ha terminado de aplicar la fuerza, respectivamente.

El factor de relajación $R$ fue expresado en porcentaje mediante la ecuación (4):

$$
R=\frac{F_{1} \square F_{2}}{F_{1}} \times 100
$$


donde $F_{1}$ es la fuerza para alcanzar la profundidad de la indentación y $F_{2}$ es la fuerza posterior de haber mantenido la profundidad de la indentación constante (Fischer-Cripps, 2004). La figura 7 muestra el ciclo de profundidad constante en el tiempo $(300 \mathrm{~nm})$ aplicado en las probetas para la determinación del factor de relajación. Las nanoindentaciones se realizaron en una superficie de $10 \mathrm{x}$ $10 \mu \mathrm{m}^{2}$, con un mínimo de 8 nanoindentaciones en la pared celular y 5 en la lamela media.

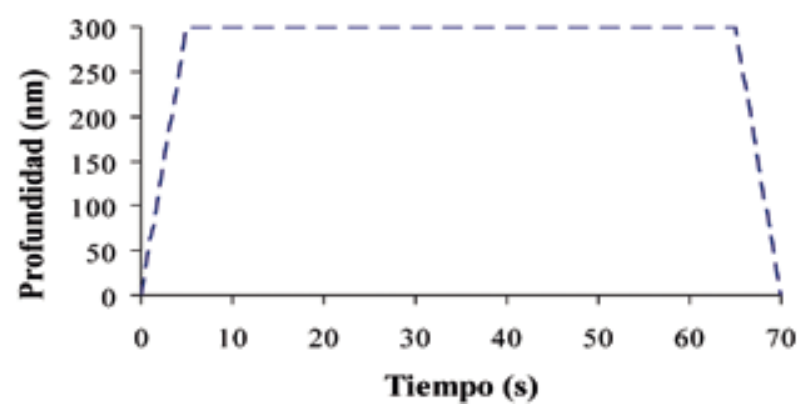

Figura 7. Ciclo de profundidad constante utilizado en la determinación del factor de relajación por medio de nanoindentaciones.

\section{Criterio de selección del tablón representativo de la probeta de tablero tensado}

La determinación del tablón representativo de la probeta de tablero tensado se basa en identificar aquel que fue notoriamente afectado por los esfuerzos de compresión perpendicular, a través de la determinación del módulo de elasticidad, dureza, factor de creep y factor de relajación de la pared celular y la lamela media de cada tablón (A, B C y D) y comparándolas con las mismas propiedades obtenidas en el tablón control (libre de cargas mecánicas).

\section{Análisis estadístico}

Un análisis de varianza (ANOVA) de dos factores fue aplicado para estudiar el efecto del tipo de madera y posición de los tablones de la probeta de tablero tensado (Tabla 2), en las propiedades nanomecánicas de la estructura celular. Se trabajó con un nivel de significancia $\alpha=0.01$. Se utilizó el procedimiento GLM ajustado a modelos lineales generales del programa SAS para el análisis de los resultados. Las comparaciones pareadas se realizaron mediante el test protected Fisher LSD.

Tabla 2. Diseño experimental general utilizado en el análisis del efecto de los tablones de la probeta de tablero tensado de pino radiata.

\begin{tabular}{|c|c|}
\hline Variables & Niveles \\
\hline \multirow{3}{*}{ Tablón } & $\mathrm{A}$ \\
& $\mathrm{B}$ \\
& $\mathrm{C}$ \\
& $\mathrm{D}$ \\
& E (control) \\
\hline \multirow{2}{*}{ Tipo de madera } & Temprana \\
& Tardía \\
\hline
\end{tabular}




\section{RESULTADOS Y DISCUSIÓN}

\section{ETAPA 1: Análisis del efecto de cargas de compresión perpendicular en los anillos de crecimiento en madera de pino radiata $y$ Eucalyptus nitens}

\section{Módulo de elasticidad en pino radiata obtenido por nanoindentaciones}

La tabla 3 muestra los valores de módulo de elasticidad $\left(E_{s}\right)$ de los anillos de crecimiento, obtenidos a diferentes cargas en la pared celular y en la lamela media en madera de pino radiata. El mayor $E_{s}$ se obtuvo en el cuarto anillo de crecimiento en células de madera tardía, a una carga de compresión de $2 \mathrm{MPa}$, con un valor de 17.8 y $8.1 \mathrm{GPa}$ para la pared celular y lamela media, respectivamente. La probeta control de pino radiata mostró un promedio de $15.3 \mathrm{GPa}$ en la pared celular. Ojeda (2010) obtuvo menores valores de $\mathrm{E}_{\mathrm{s}}$ para esta especie, con $9.1 \mathrm{GPa}$ en la pared celular. En general, el ANOVA no mostró diferencias significativas en $E_{s}$ de la pared celular, entre la madera temprana y tardía de las probetas sometidas a diferentes cargas.

Tabla 3. Estadística descriptiva de $E_{\mathrm{s}}$ de la pared celular y la lamela media en muestras de pino radiata.

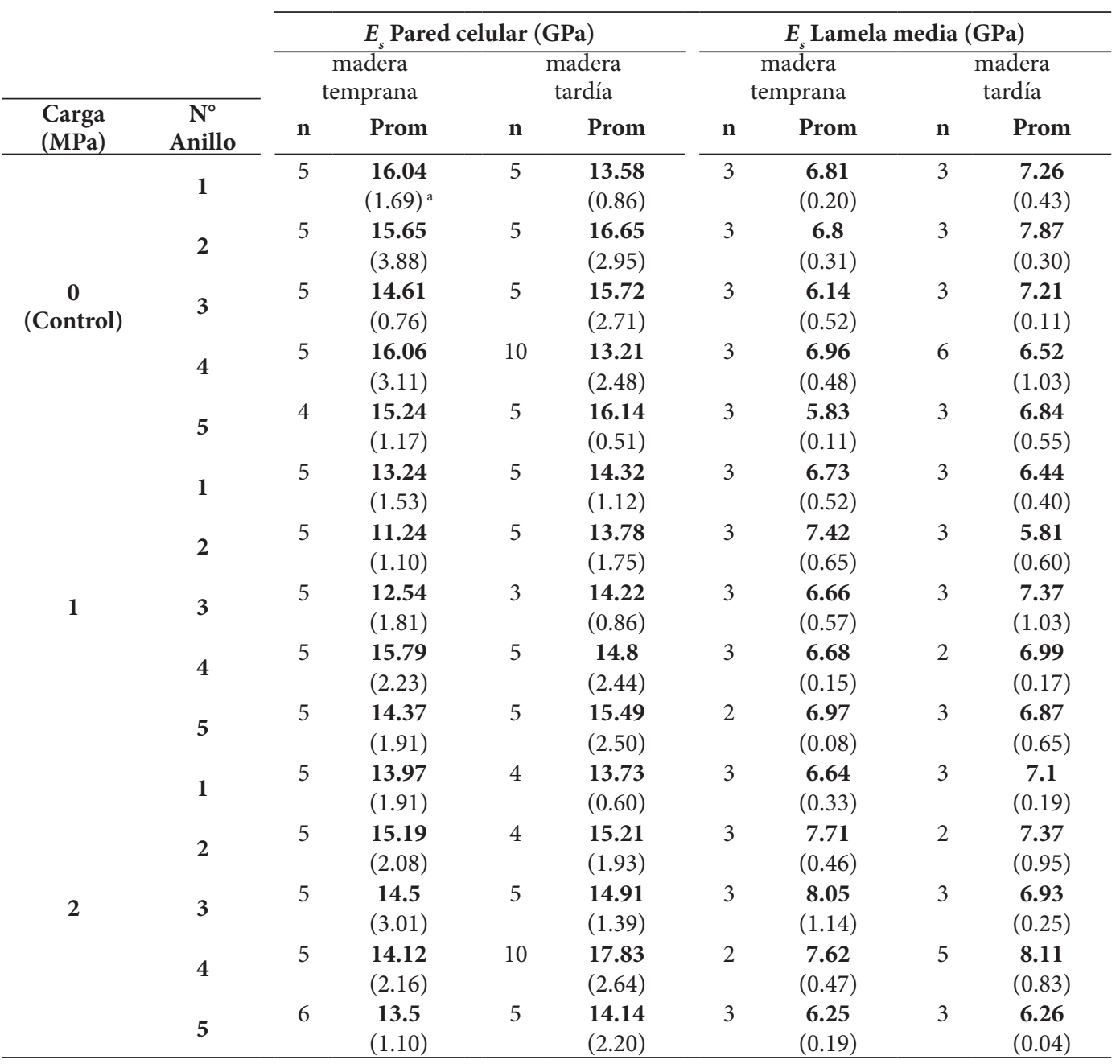

n: Número de nanoindentaciones, Prom: Promedio

a: Valores entre paréntesis, desviaciones estándar. 
Una interacción de tercer orden se produjo en la lamela media del pino radiata entre carga, anillo y tipo de madera $(\mathrm{P}=0.0037)$. Las diferencias significativas para los niveles de cargas de compresión se originaron en la lamela media de la madera tardía del segundo anillo de crecimiento, entre el control y la muestra bajo carga de $1 \mathrm{MPa}$, con valores de 7.87 y $5.81 \mathrm{GPa}$, respectivamente, y entre las muestras bajo carga de 1 y $2 \mathrm{MPa}$, con un valor de $7.37 \mathrm{GPa}$ en esta última. Para madera temprana del tercer anillo de crecimiento, estas diferencias se presentaron entre el control $\left(E_{s}=6.14 \mathrm{GPa}\right)$ y 2 $\mathrm{MPa}\left(E_{s}=8.05 \mathrm{GPa}\right)$ y entre $1 \mathrm{MPa}\left(E_{s}=6.66 \mathrm{GPa}\right)$ y $2 \mathrm{MPa}$. Para el cuarto anillo de crecimiento las diferencias significativas se encontraron en madera tardía, entre la probeta control y la probeta cargada a $2 \mathrm{MPa}$, con valores de 6.52 y $8.11 \mathrm{GPa}$. No hubo diferencias significativas de $E_{s}$ entre las cargas aplicadas para el primer y quinto anillo de crecimiento.

No hubo diferencias significativas en $E_{s}$ de la lamela media entre madera temprana y tardía para la probeta control de pino radiata, con un valor promedio de $6.8 \mathrm{GPa}$. Las diferencias entre los anillos de crecimiento se comenzaron a evidenciar cuando la madera fue sometida a mayor compresión (2 $\mathrm{MPa}$ ), con el máximo valor en la lamela media del cuarto anillo de crecimiento, con un resultado promedio de $7.9 \mathrm{GPa}$ entre madera de temprana y tardía.

\section{Dureza en pino radiata obtenida por nanoindentaciones}

La tabla 4 muestra los valores de la dureza $(\mathrm{H})$ en la pared celular y en la lamela media de los anillos de crecimiento, obtenidos a diferentes cargas en las probetas de pino radiata. La dureza en la probeta control, tanto en la pared celular como en la lamela media, alcanzó su máximo valor en madera temprana del segundo anillo de crecimiento, con resultados de 0.36 y $0.38 \mathrm{GPa}$, respectivamente. Del ANOVA $(\alpha=0.01)$, sobre un total de 156 nanoindentaciones en la pared celular, se determinó una interacción de tercer orden $\left(\mathrm{Carga}^{\star}{ }^{A}\right.$ nillo ${ }^{\star}$ Tipo de madera, $\left.\mathrm{P}<0.0001\right)$. 
Tabla 4. Estadística descriptiva de $\mathrm{H}$ de la pared celular y la lamela media de muestras de pino radiata.

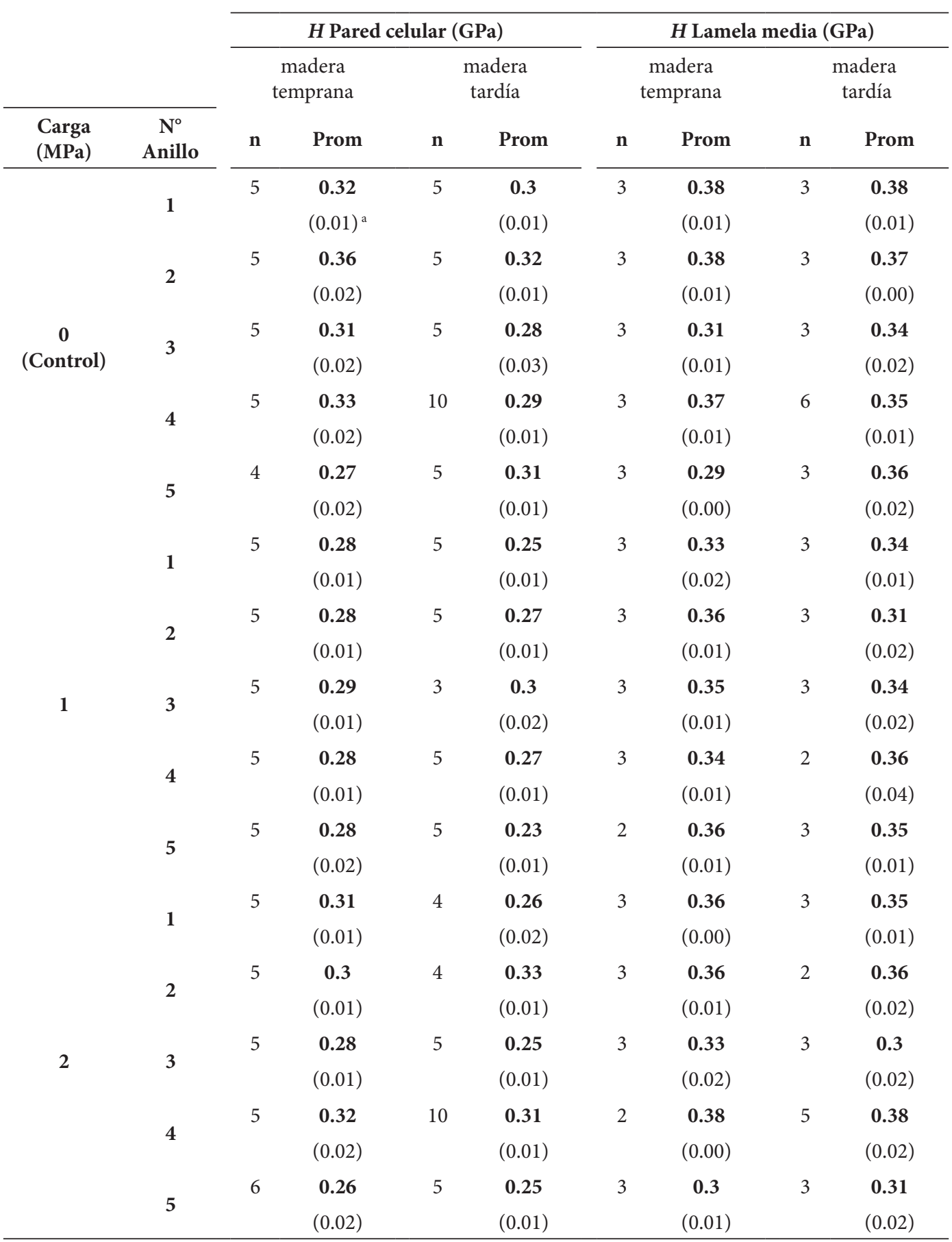

n: Número de nanoindentaciones, Prom: Promedio

a: Valores entre paréntesis, desviaciones estándar. 
Se obtuvieron diferencias significativas de $\mathrm{H}$ en la pared celular de la probeta control con las probetas cargadas a 1 y $2 \mathrm{MPa}$ y también se originaron diferencias entre estos dos niveles de carga. La probeta control mostró una mayor dureza. Se observaron diferencias en $\mathrm{H}$ de la pared celular entre madera temprana y tardía, con valores promedios de 0.30 y $0.27 \mathrm{GPa}$, respectivamente. Ojeda (2010), en su estudio de siete especies maderables chilenas, reportó $0.23 \mathrm{GPa}$ de $\mathrm{H}$ en la pared celular del pino radiata.

Una interacción de tercer orden (Carga ${ }^{\star}$ Anillo*Tipo de madera, $\left.\mathrm{P}=0.0001\right)$ se obtuvo para $\mathrm{H}$ en la lamela media del pino radiata. Las diferencias entre los niveles de cargas se mostraron en los anillos de crecimiento 1 y 2 , con mayor $\mathrm{H}$ en la probeta control $(\mathrm{H}=0.38 \mathrm{GPa})$ que las probetas bajo compresión $(\mathrm{H}=0.34 \mathrm{GPa})$. Por otro lado, se determinó que no existen diferencias significativas entre la lamela media cercana a madera temprana con la cercana a madera tardía. Esto se puede atribuir a que la lamela media está compuesta por un 65 a 75\% de lignina en coníferas, siendo la lignina un componente isotrópico (Bodig y Jayne 1993).

\section{Módulo de elasticidad en Eucalyptus nitens obtenido por nanoindentaciones}

El ANOVA no mostró diferencias significativas del módulo de elasticidad $\left(E_{s}\right)$ entre las cargas de compresión, anillos de crecimiento y tipo de madera para un nivel de confianza $\alpha=0.01$, ni en la pared celular ni en la lamela media de esta especie. No obstante, $E_{s}$ obtenido en la pared celular del Eucalyptus nitens fue en promedio $13.9 \mathrm{GPa}$. Este valor fue mayor a lo informado por Gacitúa et al. (2007) para esta misma especie, quienes determinaron un valor promedio de $10 \mathrm{GPa}$ en la pared celular. Por otro lado, Wu et al. (2009) en su estudio de 10 especies de latifoliadas determinaron un módulo de elasticidad promedio de 20.2 GPa. Esto se atribuye a la mayor densidad de estas especies, con un promedio de $0.769 \mathrm{~g} / \mathrm{cm}^{3}$, en comparación al Eucalyptus nitens que presentó una densidad de $0.484 \mathrm{~g} / \mathrm{cm}^{3}$ para las probetas en estudio. Para la lamela media del Eucalyptus nitens, $E_{s}$ muestra menores valores en comparación a la pared celular, con un resultado promedio de $8.4 \mathrm{GPa}$. Estos valores se acercan a los obtenidos por Valenzuela (2011) y Figueroa (2010), quienes determinaron un módulo de elasticidad de 7.0 y 7.5 GPa para esta especie, respectivamente.

\section{Dureza en Eucalyptus nitens obtenida por nanoindentaciones}

La dureza $(\mathrm{H})$ de la pared celular de los anillos de crecimiento del Eucalyptus nitens bajo diferentes cargas, fluctuó entre 0.27 y $0.33 \mathrm{GPa}$, y en la lamela media varió entre 0.28 y $0.39 \mathrm{GPa}$ (Tabla 5). Para $\mathrm{H}$ en la pared celular existió una interacción de segundo orden, entre la carga aplicada y los anillos de crecimiento $(\mathrm{P}=0.0004)$. Se determinó que existen diferencias significativas entre la probeta control y las que estuvieron bajo compresión. Los anillos de crecimiento 1 y 3 de las probetas bajo carga, mostraron una disminución del $12 \%$ en $\mathrm{H}$ respecto del control. Por otro lado, no se obtuvieron diferencias significativas entre los anillos de crecimiento de cada probeta (control, 1 y $2 \mathrm{MPa}$ ), con un promedio de $0.3 \mathrm{GPa}$ en la pared celular de esta especie. Para $\mathrm{H}$ en la lamela media se observaron dos interacciones de segundo orden (Anillo ${ }^{\star}$ Tipo de madera, $\mathrm{P}=0.003$; Carga ${ }^{\star}$ Anillo, $\mathrm{P}=0.001$ ). Se reveló que la lamela media cercana a células de madera tardía del primer anillo, tiene mayor $\mathrm{H}$ que la cercana a madera temprana, con valores de 0.31 y $0.35 \mathrm{GPa}$, respectivamente. El segundo anillo de crecimiento de la probeta cargada a $1 \mathrm{MPa}$ mostró $0.30 \mathrm{GPa}$ de $\mathrm{H}$ en la lamela media, mientras que en la cargada a $2 \mathrm{MPa}$, el mismo anillo de crecimiento mostró un aumento de esta propiedad a $0.39 \mathrm{GPa}$. 
Tabla 5. Estadística descriptiva de $\mathrm{H}$ de la pared celular y la lamela media de muestras de Eucalyptus nitens.

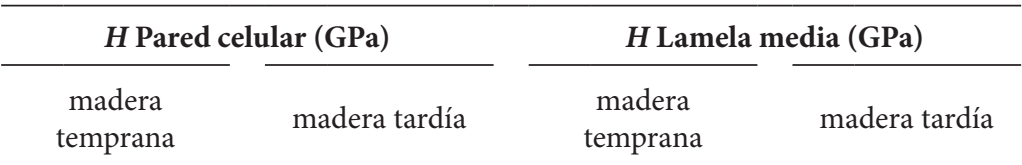

\begin{tabular}{|c|c|c|c|c|c|c|c|c|c|}
\hline $\begin{array}{l}\text { Carga } \\
(\mathrm{MPa})\end{array}$ & $\begin{array}{c}\mathbf{N}^{\circ} \\
\text { Anillo }\end{array}$ & $\mathbf{n}$ & Prom & $\mathbf{n}$ & Prom & $\mathrm{n}$ & Prom & $\mathbf{n}$ & Prom \\
\hline \multirow{6}{*}{$\begin{array}{c}\mathbf{0} \\
\text { (Control) }\end{array}$} & \multirow{2}{*}{1} & 5 & 0.33 & \multirow[t]{2}{*}{5} & 0.32 & \multirow[t]{2}{*}{3} & 0.32 & \multirow[t]{2}{*}{1} & 0.37 \\
\hline & & & $(0.01)^{\mathrm{a}}$ & & $(0.03)$ & & $(0.01)$ & & (.) \\
\hline & \multirow{2}{*}{2} & 5 & 0.31 & \multirow[t]{4}{*}{5} & 0.3 & \multirow[t]{2}{*}{3} & 0.34 & \multirow[t]{4}{*}{3} & 0.34 \\
\hline & & & $(0.01)$ & & $(0.01)$ & & $(0.02)$ & & $(0.03)$ \\
\hline & \multirow{2}{*}{3} & 5 & 0.31 & & - & \multirow[t]{2}{*}{3} & 0.36 & & - \\
\hline & & & $(0.04)$ & & & & $(0.02)$ & & \\
\hline \multirow{6}{*}{1} & \multirow{2}{*}{1} & 5 & 0.28 & \multirow[t]{2}{*}{5} & 0.29 & \multirow[t]{2}{*}{3} & 0.3 & \multirow[t]{2}{*}{3} & 0.35 \\
\hline & & & $(0.04)$ & & $(0.03)$ & & $(0.04)$ & & $(0.01)$ \\
\hline & \multirow{2}{*}{2} & 4 & 0.32 & \multirow[t]{2}{*}{4} & 0.29 & \multirow[t]{2}{*}{3} & 0.33 & \multirow[t]{2}{*}{3} & 0.28 \\
\hline & & & $(0.04)$ & & $(0.02)$ & & $(0.01)$ & & $(0.02)$ \\
\hline & \multirow{2}{*}{3} & 5 & 0.3 & & - & \multirow[t]{2}{*}{3} & 0.35 & & - \\
\hline & & & $(0.01)$ & & & & $(0.02)$ & & \\
\hline \multirow{6}{*}{2} & \multirow{2}{*}{1} & 5 & 0.27 & \multirow[t]{2}{*}{5} & 0.29 & \multirow[t]{2}{*}{3} & 0.31 & \multirow[t]{2}{*}{3} & 0.33 \\
\hline & & & $(0.03)$ & & $(0.02)$ & & $(0.01)$ & & $(0.01)$ \\
\hline & \multirow{2}{*}{2} & 5 & 0.31 & \multirow[t]{2}{*}{4} & 0.29 & \multirow[t]{2}{*}{3} & 0.38 & 2 & 0.39 \\
\hline & & & $(0.02)$ & & $(0.02)$ & & $(0.01)$ & & $(0.03)$ \\
\hline & \multirow{2}{*}{3} & 5 & 0.28 & & - & \multirow[t]{2}{*}{3} & 0.35 & & - \\
\hline & & & $(0.04)$ & & & & $(0.03)$ & & \\
\hline
\end{tabular}

n: Número de nanoindentaciones, Prom: Promedio

a: Valores entre paréntesis, desviaciones estándar.

En consecuencia, se definió que no existe una tendencia de las propiedades nanomecánicas a través de los anillos de crecimiento de las probetas de pino radiata y de Eucalyptus nitens. Sin embargo, se determinó que los niveles de compresión aplicados en las probetas de madera, alteraron las propiedades mecánicas respecto del control. Asimismo, las propiedades mecánicas a nanoescala no mostraron diferencias notorias entre la madera temprana y tardía de los anillos de crecimiento. Por lo tanto, se concluyó que no existe un anillo representativo del efecto que se produjo en la madera cuando probetas estuvieron bajo carga de compresión, más bien se determinó que es independiente analizar las propiedades mecánicas de uno u otro anillo de crecimiento, respecto de su ubicación en la sección transversal de las piezas de madera.

De este modo, para mantener un control de la extracción de muestras en piezas de madera respecto de su sección transversal, se determinó que el elemento representativo se extraerá del anillo de crecimiento más alejado a la médula para evitar la presencia de duramen en las muestras que se utilicen en la etapa 2. 


\section{ETAPA 2: Análisis a nanoescala del efecto de cargas de compresión transversal en tablones de una probeta de tablero tensado}

\section{Historial de carga de la probeta de tablero tensado}

La probeta de tablero tensado de pino radiata se comprimió en dirección normal a la fibra con una carga inicial de $0.78 \mathrm{MPa}$. Luego, se realizaron 2 retensados al mismo nivel $(0.78 \mathrm{MPa})$ a los 7 y 28 días del armado de la probeta (Figura 8), con el fin de evitar inicialmente fuertes variaciones de los niveles de compresión (Ritter 1990). El último retensado se llevó a cabo a los 65 días del armado, con una carga de compresión de $2 \mathrm{MPa}$. El nivel de compresión en el tiempo fue directamente proporcional con la humedad relativa e inversamente proporcional con la temperatura. Este comportamiento fue similar a lo informado para las especies sugi (Cryptomerica japonica D. Don), hinoki (Chamaecyparis obtusa Ende), karamatsu (Larix Leptolepis Gordon) y abeto de Douglas (Pseudotsuga menziesii (Mirb.) Franco), puesto que probetas fueron sometidas a comprensión perpendicular y a cambios cíclicos de humedad, mostrando una reducción de la compresión cuando la madera estuvo expuesta a una disminución en la humedad relativa (Zhou et al. 1999, Quenneville y Van Dalen 1994).

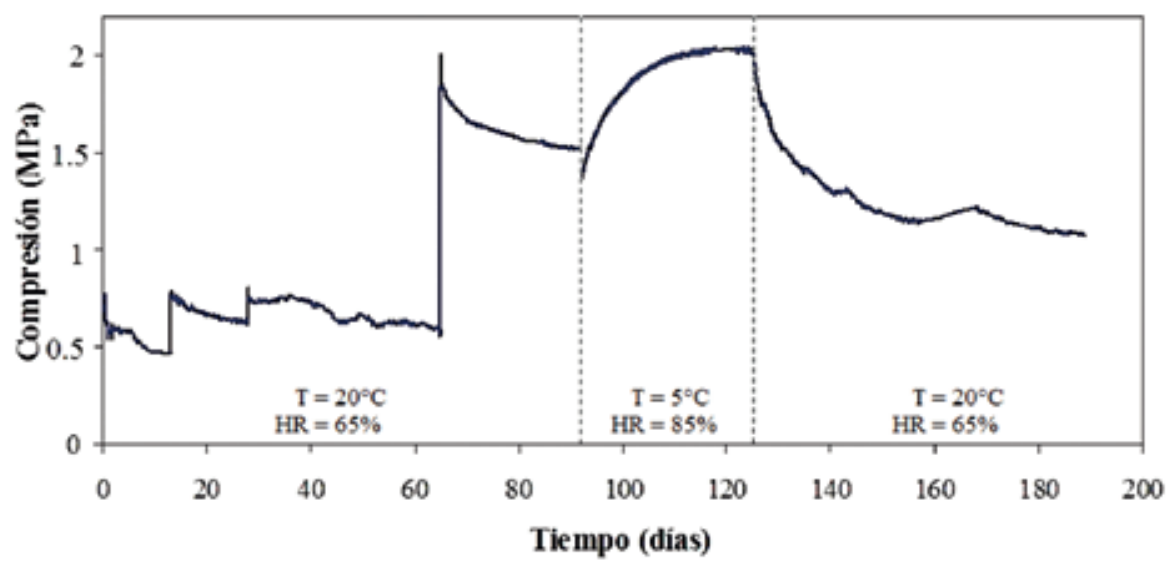

Figura 8. Historial de carga de la probeta de tablero tensado de pino radiata, indicando las condiciones ambientales a la que estuvo sometida.

\section{Módulo de Elasticidad obtenido por nanoindentaciones}

Las tablas 6 y 7 muestran los valores del módulo de elasticidad $\left(E_{s}\right)$ medidos en la pared celular y en la lamela media, respectivamente. El máximo valor de $E_{s}$ en la pared celular y en la lamela media corresponde en su mayoría al tablón D, con 16.2 GPa en la pared celular de la madera temprana y 7.3 GPa en la lamela media, en promedio entre madera temprana y tardía. Gindl et al. (2004) informaron para la conífera abeto rojo (Picea abies) similares resultados en la diferencia entre la pared celular y la lamela media, con valores de 17 y $4.5 \mathrm{GPa}$, respectivamente. De los ANOVA $(\alpha=0.01)$ se obtuvo una interacción de segundo orden entre los tablones y el tipo de madera, en la pared celular $(\mathrm{P}<0.0001)$ y en la lamela media $(\mathrm{P}=0.0004)$. El análisis mostró que las diferencias de $E_{\mathrm{s}}$ en la pared celular y en la lamela media, entre madera temprana y tardía, se produjeron en el tablón control y en el tablón C. En el tablón control, la madera temprana presentó mayor $E_{s}$ que en la madera tardía, con valores de 12.7 y $10.7 \mathrm{GPa}$ en la pared celular, respectivamente y para la lamela media se obtuvieron resultados de 7.1 y 6.6 GPa. A diferencia, el tablón C mostró lo contrario, con $11.1 \mathrm{GPa}$ en madera temprana y $13.3 \mathrm{GPa}$ en madera tardía y para la lamela media 6.1 y 7.2 GPa. Esta diferencia coincide con la investigación realizada por Wimmer et al. (1997), quienes determinaron un módulo de elasticidad para la especie de abeto rojo (Picea rubens), de 13.5 y $21 \mathrm{GPa}$ en la pared celular de madera temprana y tardía, respectivamente. Eder et al. (2009) y Cramer et al. (2005), afirmaron que el aumento de las propiedades en la madera tardía se asocia al porcentaje de celulosa y a su grado de cristalinidad, 
puesto que en esta zona (madera tardía) es mayor. No se produjeron diferencias significativas de $E_{\mathrm{s}}$ en madera tardía entre los tablones A, B, C y D, únicamente se advirtió que aumenta respecto del tablón control.

Tabla 6. Estadística descriptiva y comparación de las medias del módulo de elasticidad $\left(E_{s}\right)$ en la pared celular para la interacción entre Tablón ${ }^{\star}$ Tipo de madera.

\begin{tabular}{|c|c|c|c|c|c|c|}
\hline \multirow[b]{2}{*}{ Tablón } & \multicolumn{3}{|c|}{$E_{s}$ madera temprana (GPa) } & \multicolumn{3}{|c|}{$E_{s}$ madera tardía $(\mathrm{GPa})$} \\
\hline & $\mathbf{n}$ & Prom & Desvest & $\mathbf{n}$ & Prom & Desvest \\
\hline A & 19 & $13.6^{\star B}$ & 1.69 & 17 & $13.4^{\mathrm{B}}$ & 1.74 \\
\hline B & 18 & $13.9^{\mathrm{B}}$ & 1.48 & 16 & $13.7^{\mathrm{B}}$ & 2.14 \\
\hline $\mathrm{C}$ & 12 & $11.1^{\mathrm{CD}}$ & 0.96 & 10 & $13.3^{\text {B }}$ & 1.32 \\
\hline $\mathbf{D}$ & 10 & $16.2^{\mathrm{A}}$ & 2.42 & 12 & $13.1^{\mathrm{B}}$ & 1.95 \\
\hline E & 13 & $12.7^{\mathrm{BC}}$ & 1.79 & 17 & $10.7^{\mathrm{D}}$ & 1.81 \\
\hline
\end{tabular}

n: Número de nanoindentaciones, Prom: Promedio, Desvest: Desviación estándar *: Prueba de medias con la misma letra no son significativamente diferentes. $\alpha=0.01$

Tabla 7. Estadística descriptiva y comparación de las medias del módulo de elasticidad $\left(E_{s}\right)$ en la lamela media para la interacción entre TablónTipo de madera.

\begin{tabular}{|c|c|c|c|c|c|c|}
\hline \multirow[b]{2}{*}{ Tablón } & \multicolumn{3}{|c|}{$E_{s}$ madera temprana $(\mathrm{GPa})$} & \multicolumn{3}{|c|}{$E_{s}$ madera tardía $(\mathrm{GPa})$} \\
\hline & $\mathbf{n}$ & Prom & Desv.est & $\mathbf{n}$ & Prom & Desv.est \\
\hline A & 9 & $6.7^{\star \mathrm{BCD}}$ & 0.47 & 10 & $6.5^{\mathrm{CD}}$ & 0.65 \\
\hline B & 5 & $6.6^{\mathrm{BCD}}$ & 0.30 & 11 & $6.5^{\mathrm{D}}$ & 0.36 \\
\hline C & 7 & $6.1^{\mathrm{D}}$ & 0.42 & 5 & $7.2^{\mathrm{AB}}$ & 0.59 \\
\hline D & 9 & $7.1^{\mathrm{AB}}$ & 0.39 & 6 & $7.4^{\mathrm{A}}$ & 0.72 \\
\hline E & 12 & $7.1^{\mathrm{AB}}$ & 0.41 & 10 & $6.6^{\mathrm{CD}}$ & 0.44 \\
\hline
\end{tabular}

n: Número de nanoindentaciones, Prom: Promedio, Desv.est: Desviación estándar ${ }^{\star}$ : Prueba de medias con la misma letra no son significativamente diferentes. $\alpha=0.01$.

\section{Dureza obtenida por nanoindentaciones}

Las tablas 8 y 9 muestran los valores de la dureza $(\mathrm{H})$ en la pared celular y en la lamela media, respectivamente. Del ANOVA, se determinó una interacción de segundo orden entre tablones y tipo de madera $(\mathrm{P}=0.0004)$ para $\mathrm{H}$ en la pared celular. Los tablones A y D presentaron diferencias con el tablón control en madera temprana y tardía, en ambos casos el tablón $\mathrm{D}$ mostró una mayor dureza respecto del control.

El ANOVA $(\alpha=0.01)$ para $\mathrm{H}$ en la lamela media mostró diferencias significativas en un factor principal (Tablón, $\mathrm{P}<0.0001)$. Existió una tendencia del aumento en la dureza desde el tablón A hacia el tablón central D (Tabla 9). Este comportamiento revelaría que la resistencia mecánica del tablón A disminuyó en comparación a los tablones B, C y D. La dureza en la lamela media del tablón C no presentó diferencias significativas con el control. 
Tabla 8. Estadística descriptiva y comparación de las medias de la dureza $(H)$ en la pared celular para la interacción entre TablónTipo de madera.

\begin{tabular}{|c|c|c|c|c|c|c|}
\hline \multirow[b]{2}{*}{ Tablón } & \multicolumn{3}{|c|}{$H$ madera temprana $(\mathrm{GPa})$} & \multicolumn{3}{|c|}{$H$ madera tardía (GPa) } \\
\hline & $\mathbf{n}$ & Prom & Des.vest & $\mathbf{n}$ & Prom & Desv.est \\
\hline $\mathbf{A}$ & 19 & $0.26 * \mathrm{D}$ & 0.033 & 17 & $0.29^{\text {в }}$ & 0.022 \\
\hline B & 18 & $0.26^{\mathrm{CD}}$ & 0.016 & 16 & $0.26^{\mathrm{CD}}$ & 0.021 \\
\hline $\mathrm{C}$ & 12 & $0.28^{\mathrm{BC}}$ & 0.023 & 10 & $0.29^{\text {в }}$ & 0.026 \\
\hline D & 10 & $0.32^{\mathrm{A}}$ & 0.017 & 12 & $0.33^{\mathrm{A}}$ & 0.014 \\
\hline $\mathbf{E}$ & 13 & $0.29^{\mathrm{B}}$ & 0.024 & 17 & $0.26^{\mathrm{CD}}$ & 0.033 \\
\hline
\end{tabular}

n: Número de nanoindentaciones, Prom: Promedio, Desv.est: Desviación estándar, *: Prueba de medias con la misma letra no son significativamente diferentes. $\alpha=0.01$.

Tabla 9. Estadística descriptiva y comparación de las medias de la dureza $(H)$ en la lamela media de los tablones componentes de probetas de tablero tensado.

\begin{tabular}{|c|c|c|c|c|c|c|}
\hline \multirow[b]{2}{*}{ Tablón } & \multicolumn{3}{|c|}{$H$ madera temprana $(\mathrm{GPa})$} & \multicolumn{3}{|c|}{$H$ madera tardía $(\mathrm{GPa})$} \\
\hline & $\mathbf{n}$ & Prom & Desv.est & $\mathbf{n}$ & Prom & Desv.est \\
\hline A & 9 & $0.30^{\mathrm{C}}$ & 0.028 & 10 & $0.3^{\mathrm{C}}$ & 0.030 \\
\hline B & 5 & $0.32^{\mathrm{C}}$ & 0.007 & 11 & $0.32^{\mathrm{C}}$ & 0.018 \\
\hline $\mathrm{C}$ & 7 & $0.34^{\mathrm{B}}$ & 0.01 & 5 & $0.35^{\text {в }}$ & 0.043 \\
\hline $\mathbf{D}$ & 9 & $0.37^{\mathrm{A}}$ & 0.012 & 6 & $0.38^{\mathrm{A}}$ & 0.040 \\
\hline E & 12 & $0.34^{\text {B }}$ & 0.026 & 10 & $0.35^{\text {в }}$ & 0.014 \\
\hline
\end{tabular}

n: Número de nanoindentaciones, Prom: Promedio, Desv.est: Desviación estándar.

La figura 9 muestra la correlación entre $E_{s}$ y $\mathrm{H}$ de la estructura celular del pino radiata. Diferentes agrupaciones de datos se observan para la pared celular y la lamela media, denotando una diferencia en las propiedades mecánicas entre ellas. En la lamela media, $E_{s}$ y $\mathrm{H}$ parecieran estar correlacionadas entre sí $\left(R^{2}=0.274\right)$, en cambio, la pared celular presentó una correlación casi nula $\left(R^{2}=0.034\right)$. Estas correlaciones concuerdan con lo expuesto por Wimmer y Lucas (1997) en madera de abeto (Picea rubens), quienes establecieron que las propiedades de $E_{s}$ y $\mathrm{H}$ en la lamela media están fuertemente correlacionadas $\left(\mathrm{R}^{2}=0.55\right)$, y que no se produce tal correlación en las propiedades de la pared celular $\left(R^{2}=0.09\right)$. 


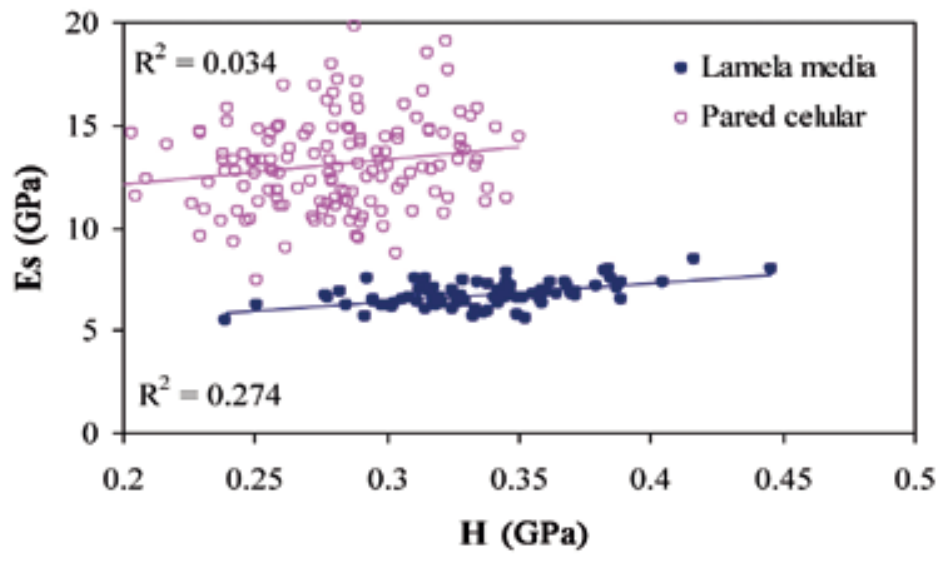

Figura 9. Correlación entre $E_{s}$ y $H$, para la pared celular y la lamela media del pino radiata.

\section{Factor de creep obtenido por nanoindentaciones}

Las tablas 10 y 11 muestran los valores del factor creep obtenidos en la pared celular y en la lamela media, respectivamente. El ANOVA estableció una interacción de segundo orden entre tablones y tipo de madera en la pared celular $(\mathrm{P}=0.0093)$. Para la lamela media el factor de creep evidenció diferencias significativas en los factores principales, tablones y tipo de madera (ambos casos con $\mathrm{P}<$ 0.0001). El tablón control mostró un mayor factor de creep en la pared celular de madera temprana en comparación a los tablones A y D. En madera tardía, se observaron diferencias entre el tablón control con los tablones A y C. Para la lamela media, la madera temprana del tablón control presentó diferencias en el factor de creep con los tablones A, C y D. Por otro lado, no se observaron diferencias significativas entre los tablones en la madera tardía.

Tabla 10. Estadística descriptiva y comparación de las medias del factor creep (C) en la pared celular para la interacción entre Tablón`Tipo de madera.

\begin{tabular}{|c|c|c|c|c|c|c|}
\hline \multirow[b]{2}{*}{ Tablón } & \multicolumn{3}{|c|}{$C$ madera temprana (\%) } & \multicolumn{3}{|c|}{$C$ madera tardía (\%) } \\
\hline & $\mathbf{n}$ & Prom & Desv.est & $\mathbf{n}$ & Prom & Desv.est \\
\hline A & 20 & $20.8^{* \mathrm{CD}}$ & 3.19 & 18 & $20.3^{\mathrm{D}}$ & 2.89 \\
\hline B & 18 & $23.6^{\mathrm{AB}}$ & 2.54 & 16 & $21.5^{\mathrm{BCD}}$ & 2.64 \\
\hline $\mathrm{C}$ & 12 & $22.3^{\mathrm{ABCD}}$ & 2.09 & 10 & $19.9^{\mathrm{D}}$ & 2.13 \\
\hline D & 10 & $20.4^{\mathrm{CD}}$ & 2.71 & 12 & $23.0^{\mathrm{ABC}}$ & 2.58 \\
\hline E & 14 & $24.0^{\mathrm{A}}$ & 3.12 & 17 & $23.3^{\mathrm{AB}}$ & 1.18 \\
\hline
\end{tabular}

n: Número de nanoindentaciones, Prom: Promedio, Desv.est: Desviación estándar,

*: Prueba de medias con la misma letra no son significativamente diferentes. $\alpha=0.01$. 
Tabla 11. Estadística descriptiva y comparación de las medias del factor creep $(C)$ en la lamela media para la interacción entre Tablón`Tipo de madera.

\begin{tabular}{|c|c|c|c|c|c|c|}
\hline \multirow[b]{2}{*}{ Tablón } & \multicolumn{3}{|c|}{$C$ madera temprana (\%) } & \multicolumn{3}{|c|}{$C$ madera tardía (\%) } \\
\hline & $\mathbf{n}$ & Prom & Desv.est & $\mathbf{n}$ & Prom & Desv.est \\
\hline A & 11 & $21.7 \star \mathrm{BC}$ & 1.5 & 10 & $24.2^{\mathrm{A}}$ & 1.89 \\
\hline B & 5 & $24.1^{\mathrm{A}}$ & 0.81 & 11 & $24.6^{\mathrm{A}}$ & 0.84 \\
\hline $\mathrm{C}$ & 7 & $21.9^{\mathrm{BC}}$ & 0.50 & 5 & $23.7^{\mathrm{A}}$ & 1.54 \\
\hline D & 9 & $20.6^{\text {B }}$ & 1.18 & 7 & $23.4^{\mathrm{A}}$ & 1.58 \\
\hline $\mathbf{E}$ & 11 & $23.7^{\mathrm{A}}$ & 1.36 & 10 & $25.0^{\mathrm{A}}$ & 1.55 \\
\hline
\end{tabular}

n: Número de nanoindentaciones, Prom: Promedio, Desv.est: Desviación estándar

*: Prueba de medias con la misma letra no son significativamente diferentes. $\alpha=0.01$.

\section{Factor de relajación obtenido por nanoindentaciones}

Los resultados del factor de relajación en la pared celular y en la lamela media se presentan en las tablas 12 y 13 . El valor mínimo para la pared celular y para la lamela media fue 40.6 y $37.4 \%$, respectivamente, y los máximos fueron 50.3 y 49.3\%. Para ambos casos, se determinó una interacción de segundo orden entre los tablones y el tipo de madera $(\mathrm{P}=0.0091$ en pared celular, $\mathrm{P}<0.0001$ en lamela media). El tablón control mostró un aumento del 9.3\% en el factor de relajación de la pared celular de madera temprana, en comparación al tablón D, y una reducción del 6.2\% en madera tardía. De las mediciones realizadas en la lamela media, se determinó que la madera temprana del tablón control mostró un mayor factor de relajación respecto a los tablones $\mathrm{B}, \mathrm{C}$ y D, mientras que en madera tardía sólo presentó una menor relajación que el tablón A. Por otro lado, el tablón control mostró diferencias significativas entre madera temprana y tardía en la pared celular y en lamela media, evidenciando una mayor relajación en la madera temprana.

Tabla 12. Estadística descriptiva y comparación de las medias del factor de relajación $(R)$ en la pared celular para la interacción entre Tablón`Tipo de madera.

\begin{tabular}{|c|c|c|c|c|c|c|}
\hline \multirow[b]{2}{*}{ Tablón } & \multicolumn{3}{|c|}{$R$ madera temprana (\%) } & \multicolumn{3}{|c|}{$R$ madera tardía (\%) } \\
\hline & $\mathbf{n}$ & Prom & Desv.est & $\mathbf{n}$ & Prom & Desv.est \\
\hline A & 10 & 45.7 *ABC & 8.02 & 11 & $47.0^{\mathrm{ABC}}$ & 4.84 \\
\hline B & 11 & $44.6^{\mathrm{ABC}}$ & 4.13 & 11 & $42.1^{\mathrm{BC}}$ & 7.23 \\
\hline $\mathrm{C}$ & 12 & $46.6^{\mathrm{ABC}}$ & 6.43 & 13 & $50.3^{\mathrm{A}}$ & 6.11 \\
\hline D & 10 & $40.8^{\mathrm{C}}$ & 3.42 & 7 & $46.8^{\mathrm{ABC}}$ & 9.97 \\
\hline E & 6 & $50.1^{\mathrm{AB}}$ & 7.12 & 9 & $40.6^{\mathrm{C}}$ & 6.86 \\
\hline
\end{tabular}

n: Número de nanoindentaciones, Prom: Promedio, Desv.est: Desviación estándar

${ }^{*}$ : Prueba de medias con la misma letra no son significativamente diferentes. $\alpha=0.01$. 
Tabla 13. Estadística descriptiva y comparación de las medias del factor de relajación $(R)$ en la lamela media para la interacción entre TablónTipo de madera.

\begin{tabular}{|c|c|c|c|c|c|c|}
\hline \multirow[b]{2}{*}{ Tablón } & \multicolumn{3}{|c|}{$R$ madera temprana $(\%)$} & \multicolumn{3}{|c|}{$R$ madera tardía (\%) } \\
\hline & $\mathbf{n}$ & Prom & Desv.est & $\mathbf{n}$ & Prom & Desv.est \\
\hline A & 3 & $43.5^{\mathrm{ABC}}$ & 1.10 & 5 & $46.5^{\mathrm{A}}$ & 0.95 \\
\hline B & 6 & $38.9^{\mathrm{DE}}$ & 2.58 & 8 & $40.9^{\mathrm{CD}}$ & 2.58 \\
\hline $\mathrm{C}$ & 7 & $41.5^{\mathrm{BCD}}$ & 3.05 & 5 & $40.6^{\mathrm{CD}}$ & 1.90 \\
\hline $\mathbf{D}$ & 7 & $37.4^{\mathrm{E}}$ & 0.99 & 6 & $44.0^{\mathrm{AB}}$ & 1.99 \\
\hline E & 3 & $46.3^{\mathrm{A}}$ & 1.25 & 7 & $42.3^{\mathrm{BC}}$ & 1.36 \\
\hline
\end{tabular}

n: Número de nanoindentaciones, Prom: Promedio, Desv.est: Desviación estándar

*: Prueba de medias con la misma letra no son significativamente diferentes. $\alpha=0.01$.

En base a los resultados obtenidos de las propiedades nanomecánicas, los tablones que presentaron mayor diferencia en relación al control, fueron el A y el D. Sin embargo, el tablón D presentó una diferencia en la dureza respecto de los otros tablones, que evidencia la acción de las cargas externas como una tendencia al incremento de esta propiedad desde el borde de la probeta tensada hacia el centro. Por lo tanto, el tablón D permitiría explorar el comportamiento de una probeta de tablero tensado. En cuanto al tipo de madera, la variabilidad no presentó una tendencia clara, por lo tanto, los post-estudios de las propiedades nanomecánicas se centran en la madera temprana. Esto permite mayor precisión en las mediciones con nanoindentaciones, ya que el ancho de la madera temprana es mayor a la madera tardía.

Lo anterior, señala que el elemento representativo para los tablones de pino radiata se definió en el tablón D (central) para estudios referidos a probetas de tablero tensado.

En consecuencia, el elemento de volumen representativo de las probetas de tableros tensados corresponde a un cubo de $3 \mathrm{~mm}$ por lado, localizado en el tablón $\mathrm{D}$, y en la madera temprana del anillo de crecimiento más alejado de la médula.

\section{CONCLUSIONES}

Se definió un elemento de volumen representativo mediante el análisis y la caracterización de las propiedades nanomecánicas en la estructura celular, bajo condiciones de carga de compresión perpendicular, en madera de Pinus radiata y Eucalyptus nitens. Éste se localizó en madera temprana, del anillo de crecimiento más alejado a la médula en la sección transversal del tablón central (D) en una probeta de tablero tensado. Se determinó que no existen diferencias mayormente significativas de las propiedades de módulo de elasticidad y dureza, medidas en la pared celular y en la lamela media, entre los anillos de crecimiento de la sección transversal en piezas de madera. Como también, las propiedades nanomecánicas no mostraron diferencias entre madera temprana y madera tardía, estableciéndose que no existe un efecto evidente de las cargas de compresión aplicadas a los tablones en el tipo de madera (temprana y tardía). Por último, para la lamela media existió una correlación positiva entre el módulo de elasticidad y la dureza, es decir, al incrementarse el módulo de elasticidad, la dureza aumentó. Sin embargo, no existió una buena correlación para las propiedades medidas en la pared celular. 


\section{BIBLIOGRAFIA}

Astley, R.; Harrington, J.; Stol, K. 1997. Mechanical modelling of wood microstructure, an engineering approach. IPENZ Transactions 24(1):21-29.

Bodig, J.; Jayne, B. 1993. Mechanics of wood and wood composites. Krieger, Florida. USA. 712 p.

BS. British Standards Institution. 2003. Structural timber - Strength classes. BS EN 338. London, UK. 14 p.

Cramer, S.; Kretschmann, D.; Lakes, R.; Schmidt, T. 2005. Earlywood and latewood elastic properties in loblolly pine. Holzforschung 59(5):531-538.

Diaz-vaz, J.E. 2003. Anatomía de la madera. Marisa Cúneo Ediciones, Valdivia. Chile. 151 p.

Eder, M.; Jungnikl, K.; Burgert, I. 2009. A close-up view of wood structure and properties across a growth ring of Norway spruce (Picea abies [L] Karst.). Trees 23(1):79-84.

Figueroa, E. 2010. Relación entre propiedades a nanoescala y a mesoescala para madera de Eucalyptus nitens. Seminario de Título. Departamento de Ingeniería en Maderas, Universidad del BíoBío. Concepción. Chile. 73 p.

Figueroa, M. 2011. Análisis del comportamiento reológico y termohigromecánico en tableros tensados de madera para puentes a condiciones ambientales. Tesis de Magíster en Ciencia y Tecnología de la Madera. Departamento de Ingeniería en Maderas, Universidad del Bío-Bío. Concepción. Chile. 158 p.

Fischer-Cripps, A. C. 2004. Nanoindentation. Springer-Verlag, New York. USA. 266 p.

Fritz, A. 2004. Manual La Construcción de Viviendas en Madera. Santiago, Chile. CORMA. 643 p.

Gacitúa, W.; Ballerini, A.; Lasserre, J. P.; Bahr, D. 2007. Nanoindentaciones y ultraestructura en madera de Eucalyptus nitens con micro y meso Grietas. Maderas. Ciencia y tecnología 9(3):259-270.

Gindl, W.; Gupta, H.S.; Schöberl, T.; Lichtenegger, H.C.; Fratzl, P. 2004. Mechanical properties of spruce wood cell walls by nanoindentation. Applied Physics, A: Materials Science \& Processing 79(8):2069-2073.

INFOR. 2004. Eucalyptus nitens en Chile: Primera monografía. Informe técnico $\mathrm{N}^{\circ} 164$. Instituto forestal. Valdivia, Chile. $143 \mathrm{p}$.

INFOR. 2010. Anuario forestal. Boletín estadístico Nº128. Instituto forestal. Valdivia, Chile. 121 p.

Muñoz, J. 2002. Tableros de puentes de madera postensados transversalmente. Memoria de título. Departamento de Ingeniería Civil, Universidad de Concepción. Concepción. Chile. 155 p.

Navi, P.; Heger, F. 2005. Comportement thermo-hydromécanique du bois: Applications technologiques et dans les structures. Presses polytechniques et universitaires romandes. Lausana, Suiza. $298 \mathrm{p}$. 
Ojeda, E. 2010. Caracterización nanomecánica de la madera temprana de siete especies maderables chilenas. Seminario de Título. Departamento de Ingeniería en Maderas, Universidad del BíoBío. Concepción. Chile. 80 p.

Panshin, A. J.; De Zeeuw, C. 1980. Textbook of wood technology. Structure, identification, properties and uses of the commercial woods of the United States and Canada. 4th ed. McGraw-Hill, Nueva York. USA. 722 p.

Quenneville, P.; Van Dalen, K. 1994. Relaxation behaviour of prestressed wood assemblies. Part 1: Experimental study. The Canadian Journal of Civil Engineering 21(5):736-743.

Ritter, M.A. 1990. Timber Bridges: Design, construction, inspection and maintenance. Washington, DC. USA. 944 p.

Salmén, L. 2004. Micromechanical understanding of the cell-wall structure. C. R. Biologies 327:873-880.

Ugalde, G. 2004. Diseño y Construcción Tablero de Madera Tensado para el Puente Cautín. Memoria de título. Departamento de Ingeniería Civil, Universidad de Concepción. Concepción, Chile. $183 \mathrm{p}$.

Valavala, P.; Odegard, G.; Aifantis, E. 2009. Influence of representative volume element size on predicted elastic properties of polymer materials. Modelling and Simulation in Materials Science and Engineering 17(4):1-15.

Valenzuela, P. 2011. Fracturas, propiedades nanomecánicas y anatomía de familias de Eucalyptus nitens. Tesis de Magíster en Ciencia y Tecnología de la Madera. Departamento Ingeniería en Maderas. Universidad del Bío-Bío. Concepción. Chile. 85 p.

Wimmer, R.; Lucas, B. N.; Tsui, T. Y.; Oliver, W. C. 1997. Longitudinal hardness and Young's modulus of spruce tracheid secondary walls using nanoindentation technique. Wood Science and Technology 31(2):131-141.

Wimmer, R; Lucas, B. 1997. Comparing mechanical properties of secondary wall and cell middle lamella in spruce wood. IAWA Journal 18(1):77-88.

Wu, Y.; Wang, S.; Zhou, D.; Xing, C.; Zhang, Y. 2009. Use of nanoindentation and silviscan to determine the mechanical properties of 10 hardwood species. Wood and Fiber Science 41(1):64-73.

Zhou, Y.; Fushitani, M.; Kubo, T.; Ozawa, M. 1999. Bending creep behavior of wood under cyclic moisture changes. Journal Wood Science 45(2):113-119. 
